\title{
Effect of grain shape on quasi-static fluid-fluid displacement in porous media
}

\author{
Zhongzheng Wang ${ }^{1,2}$, Jean-Michel Pereira ${ }^{2}$, and Yixiang Gan ${ }^{1,3}$ \\ ${ }^{1}$ School of Civil Engineering, The University of Sydney, NSW 2006, Australia. \\ ${ }^{2}$ Navier, Ecole des Ponts, Univ Gustave Eiffel, CNRS, Marne-la-Vallée, France. \\ ${ }^{3}$ The University of Sydney Nano Institute (Sydney Nano), The University of Sydney, NSW 2006, \\ Australia
}

\section{Key Points:}

- A novel pore network model algorithm is developed to probe the effect of grain shape on multiphase displacement in porous media.

- Systematic simulations are conducted using the proposed algorithm across a wide range of wetting conditions and particle shapes.

- Through analyzing various metrics during displacement, the results highlight the profound influence of particle shape on multiphase flow.

Corresponding author: Yixiang Gan, yixiang.gan@sydney.edu.au 


\begin{abstract}
We study how grain shapes impact multiphase flow in porous media in the quasi-static regime using an extended pore-network model. The algorithm allows the explicit determination of different types of pore-scale instabilities and tracks the interface motion during fluid-fluid displacement process. It also includes the volume capacitance model, such that both the evolution of capillary pressure signal and sizes of Haines jumps can be captured. Further, it considers the pinning of menisci at sharp edges of grains, through which the distribution of effective contact angles can be obtained. Simulations are carried out across a wide range of wetting conditions for different particle shapes. Our results show that the effective contact angle distribution during displacement widens as the grain becomes more angular, which consequently modifies the macroscopic fluid invasion morphology. By analyzing various characteristic metrics during displacement, including capillary pressure signal, Haines jump size distribution, and fractal dimension, our results highlight the profound influence of particle shape on the multiphase flow.
\end{abstract}

\title{
1 Introduction
}

Fluid-fluid displacement in porous media is a common phenomenon encountered in a wide range of natural and industrial processes, such as water infiltration into soil (Lipiec et al., 2006), carbon sequestration (Szulczewski et al., 2012; Matter et al., 2016), enhanced oil recovery (Lake et al., 2014; M. Blunt et al., 1993), and remediation of contamination in aquifer systems (Nadim et al., 2000). As indicated by the pioneering works by Lenormand et al (Lenormand et al., 1988; Lenormand \& Zarcone, 1989), the multiphase displacement patterns strongly depend on the capillary number (i.e., relative strength of viscous force to capillary force) and the viscosity ratio of the two fluids, and a phase diagram including capillary fingering, viscous fingering, and stable displacement was presented. Since then, extensive efforts have been devoted to further investigation of how fluid properties, flow conditions, and topological characteristics of the porous media modify the invasion morphology (Yortsos et al., 1997; Armstrong et al., 2014; Rabbani et al., 2018; Holtzman, 2016; Wang et al., 2019; Hu et al., 2019; Ju et al., 2020; Xu et al., 2014). Specifically, both numerical and experimental works have revealed the profound influence of wettability (i.e., contact angle) in two phase flows (Crisp \& Thorpe, 1948; Purcell, 1950; Mason \& Morrow, 1994; Cieplak \& Robbins, 1990; Trojer et al., 2015; Jung et al., 2016; Wang et al., 2019, 2020; Holtzman \& Segre, 2015; Zhao et al., 2016; Ran et al., 2018; Primkulov et al., 2018). However, the effective contact angle, as one of the key controlling factors, is often unknown prior to the displacement process due to the complex geometry of pore space. Even for chemically homogeneous porous media, a wide distribution of contact angles have been observed due to roughness and pinning of menisci at sharp edges (AlRatrout et al., 2018; M. J. Blunt et al., 2019, 2021). Therefore, it is important to understand how particle shape affects the effective contact angles, which can consequently alter the pore-scale instability events and macroscopic invasion morphology (Cieplak \& Robbins, 1990; Holtzman \& Segre, 2015; Geistlinger \& Zulfiqar, n.d.; Zulfiqar et al., n.d.; AlRatrout et al., 2018).

In the quasi-static regime of multiphase flow where capillary force dominates the displacement, various numerical approaches have been developed to supplement experiments, including Navier-Stokes equation solvers and pore-network models. The methods of the latter category have been successfully applied in investigation of macroscopic invasion patterns due to significantly less computational cost (M. J. Blunt, 1998, 2001; Cieplak \& Robbins, 1988, 1990; Holtzman \& Segre, 2015; Holtzman, 2016; Primkulov et al., 2018; Hu et al., 2019). A subclass of pore-network models, the interface tracking algorithm, initially proposed by Cieplak and Robbins (Cieplak \& Robbins, 1988, 1990) and recently extended by Primkulov et al. (Primkulov et al., 2018) for consideration of corner flow, has been found successful in reproducing multiphase displacement experiments in Hele-Shaw cells (Chapuis et al., 2008; Trojer et al., 2015; Holtzman \& Segre, 
2015; Holtzman, 2016; Zhao et al., 2016; Ran et al., 2018). This method captures the pore-scale invasion mechanisms by taking into account the local pore geometry, including the cooperative pore-filling event, which stabilizes the invasion during imbibition (Cieplak \& Robbins, 1988, 1990; Holtzman \& Segre, 2015). However, up to now, this type of porenetwork models is applicable to perfectly spherical particles, whereas grains with irregular shapes are prevalent in natural systems such as sand packs, and solid walls characterized by surface with sharp edges due to manufacture limitations are encountered in microfluidics. These non-smooth surfaces can often lead to pinning of menisci during displacement process, which results in effective contact angles deviating from the intrinsic one, altering the capillary resistance at local pore/throat. It is worth noting that surface roughness has also proven influential on the effective contact angle during fluidfluid displacement process (Mehmani et al., 2019; Zulfiqar et al., n.d.; AlRatrout et al., 2018; Chen et al., 2018). One option to distinguish the impact from particle shape and roughness on contact angle is whether the effect is transient. Due to surface roughness, the effective contact angle can change as the liquid fills the grooves of the surface, leading to a time-dependent behaviour of contact angle. This phenomenon has been extensively observed in experiments (Mishra et al., 2016; Moulinet \& Bartolo, 2007; Sbragaglia et al., 2007; Seo et al., 2018; Papadopoulos et al., 2013), where the effective contact angle could be described by the Wenzel or Cassie-Baxter model, depending on the wetting states (Wenzel, 1936; Cassie \& Baxter, 1944; Marmur, 2003; Gao \& Yan, 2009). On the other hand, the pinning of the meniscus at sharp edges (Gibbs, 1961; Oliver et al., 1977), is a thermodynamically stable configuration. In the current study, we thus focus on the latter phenomenon in the quasi-static displacement process.

Here, we develop an extended pore-network model (called EPONM) to probe the effect of particle shape on quasi-static fluid-fluid displacement. The model incorporates the explicit determination of basic pore-scale instabilities based on the work of Cieplak and Robbins (Cieplak \& Robbins, 1988, 1990). It also includes the volume capacitance model (Måløy et al., 1992; Furuberg et al., 1996), which allows us to capture both the evolution of capillary pressure signal and sizes of Haines jumps. Different from the original algorithm where the volume capacitance is a prescribed constant (Måløy et al., 1992; Furuberg et al., 1996), it is calculated based on local pore geometries without extra assumptions. More importantly, the sharp edge pinning effect is added to consider the pinning of the menisci (Gibbs, 1961; Oliver et al., 1977). Our results for different grain shapes indicate that increase in angularity leads to wider distribution of contact angles, which explains the observed greater fluctuations in capillary pressure. Besides, it is found that comparing with more spherical particles, the mean capillary pressure for angular grains is greater in drainage whereas smaller in imbibition. We quantify and analyze the correlation between grain shape and size distribution of Haines jumps, interfacial length, and fractal dimension across a wide range of wetting conditions. The implications of our findings are then discussed.

\section{Extended Pore-Network Model}

To model the 2D flow patterns observed in Hele-Shaw cells filled with vertical posts (Zhao et al., 2016; Trojer et al., 2015; Hu et al., 2019; Primkulov et al., 2018) with controlled particle shapes, the porous medium is represented by polygons (instead of circles in past studies) placed on two-dimensional triangular lattice. The invading fluid is injected from the center of the simulation domain. Based on a purely geometrical extension of Young-Dupre equation (Gibbs, 1961; Oliver et al., 1977), the equilibrium state of effective contact angle $\theta$ measured within the invading fluid at the triple line follows:

$$
\theta_{0} \leq \theta \leq \theta_{0}+\left(180^{\circ}-\alpha\right)
$$

where $\theta_{0}$ and $\alpha$ are the intrinsic contact angle and the angle subtended by the two surfaces forming the edge, respectively (Fig. 1(A)). Since in this work the focus is placed 

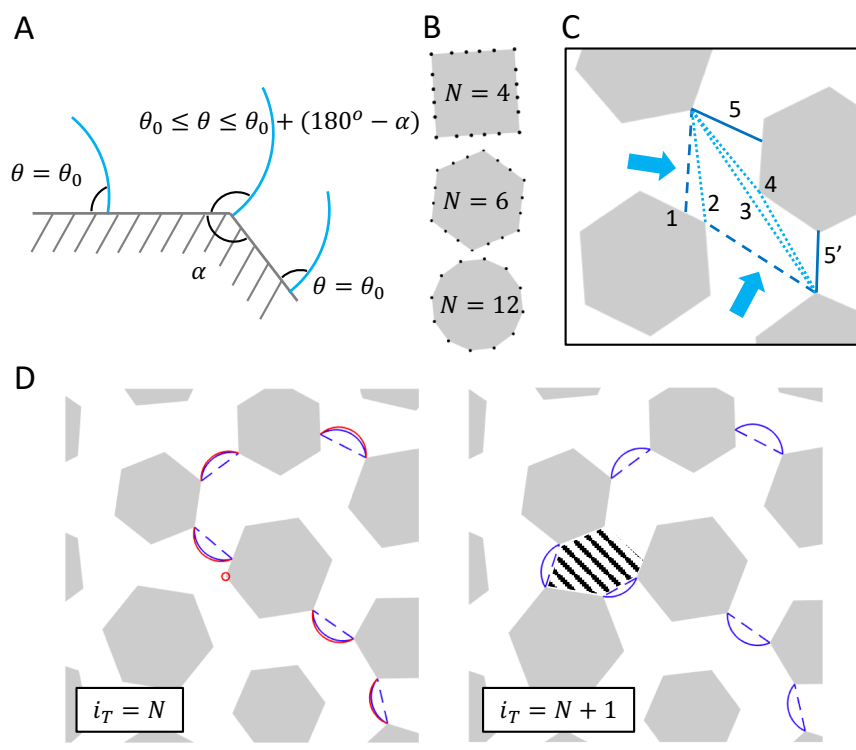

Figure 1. (A) Pinning of menisci at corners leads to greater effective contact angle. (B) Investigated grain shapes in this work with different number of edges $N$. The black dots represent the mesh points. (C) Schematic showing different invasion types. $\left\{1-2,2-3,3-4,4-5\left(5^{\prime}\right)\right\}$ correspond to \{unpin, overlap, touch, unpin \} events, respectively. Blue arrows mark the movement direction of menisci. (D) Snapshots of invasion morphology at two consecutive steps. Blue solid lines represent menisci after relaxation of previous step. Red solid lines represent menisci at the critical state (in this case an unpin event marked by the red circle will take place). The shaded area is invaded, accompanied by retraction of menisci from red lines at $i_{T}=N$ to blue lines at $i_{T}=N+1$. 


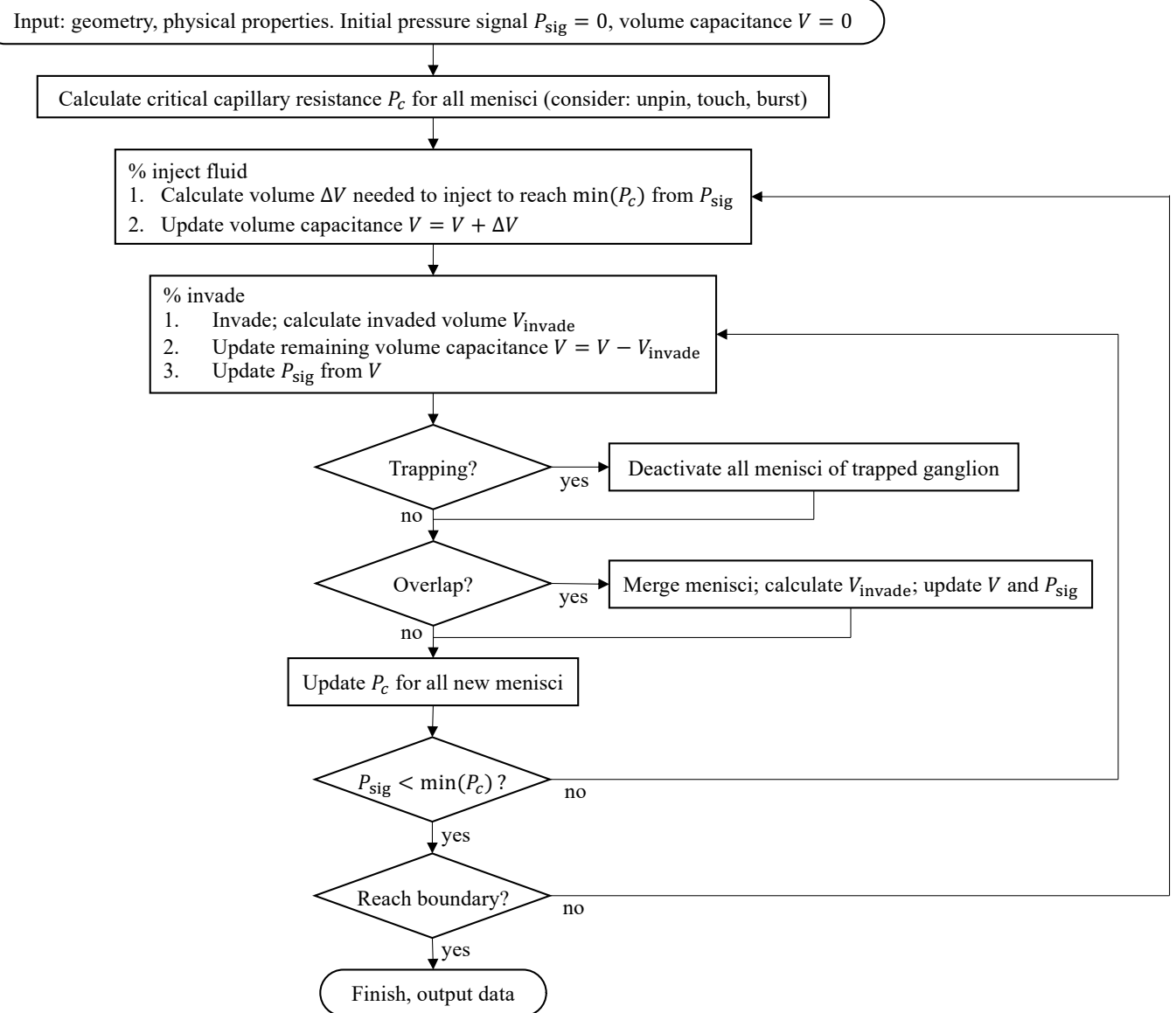

Figure 2. Flow chart of the pore-network model.

on the regime of quasi-static displacement, the advancement of liquid front is governed by capillary force, and the viscous effect is ignored.

In the framework of interface tracking algorithm, the menisci move forward through two types of advancements: (1) pressure-driven events and (2) spontaneous events (relaxation). With a constant injection velocity boundary condition, the capillary pressure builds up accompanied by change in shapes in menisci, until either the meniscus jumps towards the next mesh point due to the local contact angle being greater than the upper bound according to Eqn. 1 (unpin event), or the meniscus touches other grain, forming two new menisci (touch event). Regarding the fineness of the mesh, the number of mesh points needed per edge is denoted by $M=E / N$, with $E$ the effective number of mesh points per grain and $N$ the number of edges a grain has. It is found that $E=24$ is sufficient for the studied grain shapes, leading to the corresponding number of mesh points per edge $M=6,4,2$ for square, hexagon, and dodecagon, respectively (Fig. 1(B)). This is verified by checking both the macroscopic invasion morphology and pore-scale instability events (see Supporting Information for the mesh sensitivity test). After each pressure-driven event, the newly invaded area is subtracted from the total volume capacitance (Måløy et al., 1992; Furuberg et al., 1996) (area between red and blue-dashed lines in Fig. 1(D)), from which the pressure within the invading fluid is updated according to the remaining volume capacitance, i.e., redistributing the total volume back to each active meniscus assuming all are at thermodynamic equilibrium, i.e., all have the same curvature. Then, potential overlap event and further advancement events including un- 
pin and/or touch are checked and executed. This process is carried out until the smallest capillary resistance at the invasion front is greater than the remaining pressure within the invading fluid. Note that after each time step, trapping is checked and all menisci that belong to trapped regions are deactivated to prevent any further movement. Fig. 1(C) shows the schematic of several advancement steps initialized by a pressure-driven unpin event. Fig. 1(D) shows local snapshot of invasion morphology at two consecutive time steps. The blue-solid lines denote the menisci shape after the previous relaxation step, and red-solid lines denote the menisci shape associated with the next minimum critical capillary pressure. The flowchart of the algorithm is shown in Fig. 2. More details on the algorithm, such as the calculation of critical capillary pressure, determination of instability modes, and conversion between pressure and volume can be found in Supporting Information.

To investigate the effect of particle shapes, squares, hexagons, and dodecagons were chosen as representative grains with decreasing angularity (Fig. 1(B)). The 1-by-1 rectangular simulation domain contains in total 7520 particles placed on triangular lattice, corresponding to 94 columns of 80 vertically aligned grains with constant porosity of $0.5912 \pm$ 0.0005 (see a sample packing structure in Fig. 3(A)). Disorder is introduced by (i) inducing 10 percent variation in particle size with a uniform distribution and (ii) random rotation of particles in $\left[0^{\circ}, 360^{\circ}\right)$. The capillary pressure signal, total injected fluid volume (area in 2D), and size of Haines jump are recorded at each step, until the invasion front reaches the boundary. Simulations were carried out for each particle shape with five randomly generated porous media under contact angles ranging from $45^{\circ}$ to $165^{\circ}$ with $15^{\circ}$ increment. For displacement processes with contact angles below $45^{\circ}$, the 3D phenomenon corner flow starts to appear(Zhao et al., 2016; Primkulov et al., 2018), which is currently not captured in the model, and thus those processes are not covered in the present work.

A typical evolution of the invasion front until breakthrough for hexagonal grains with an intrinsic contact angle of $\theta_{0}=120^{\circ}$ is shown in Fig. 3(A). Crimson represents the initial stage whereas yellow indicates the late times. The displacement pattern contains rather ramified structures with significant trapping, which represents the capillary fingering in drainage. Fig. 3(B-i) shows the evolution of the dimensionless capillary pressure calculated as the curvature $P_{c}^{*}=1 / r^{*}$, with $r^{*}$ being the radius of the meniscus. The pressure-driven events and spontaneous events are marked by red circles and yellow cross, respectively. Clearly, multiple spontaneous events can take place following a pressure-driven event, which is a manifestation of a Haines jump. In the limit of vanishing capillary number, Haines jump can be regarded as effectively instantaneous compared with the speed of fluid injection at the inlet. Thus, after conversion from step into time, which is expressed as volume of injected area normalized by the average pore area, Fig. 3(B-ii) shows the pressure signal as a function of $A_{\mathrm{inj}}^{*}$. The $P_{c}^{*}$ values at same invasion progress are marked by the same number in Fig. 3(B-i) and Fig. 3(B-ii). Specifically, the processes marked by (1-2) and (2-3) represent a fast Haines jump accompanied by drop in pressure, and slow injection of invading fluid until the next critical $P_{c}^{*}$ is reached, respectively. Fig. 3(B-iii) shows $P_{c}^{*}$ signal for the whole simulation. Similar pressure signal signatures in a stick-slip manner have been observed in experiments at quasi-static condition (Måløy et al., 1992; Furuberg et al., 1996; Moura et al., 2020).

\section{Results and Discussion}

The phase diagram of the displacement patterns across a wide range of contact angles for different particle shapes is shown in Fig.4(A). The invasion morphology for medium with dodecagons at $\theta_{0}=45^{\circ}$ is compact with rather smooth front. This hexagonal shape is a direct result of the grain placement in the triangular lattice, which has been observed in previous study (Lenormand, 1990; Holtzman, 2016). However, with increase in angularity, despite the displacement pattern is still relatively stable without trapping, the 

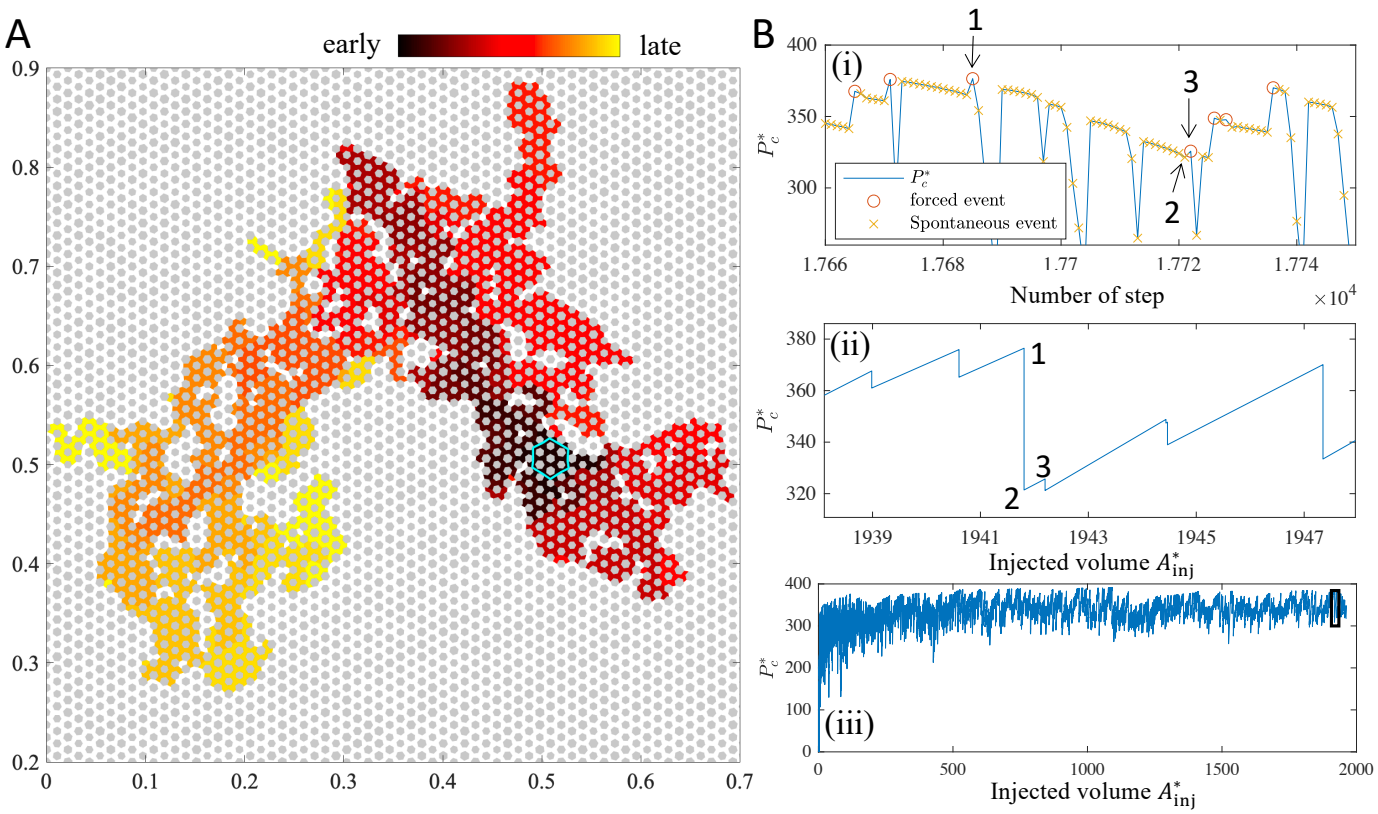

Figure 3. (A) Invasion morphology with hexagon grains (Number of edges $N=6$ ) and $\theta_{0}=120^{\circ}$. The color represents displacement patterns at different steps. The initial positions of menisci are shown as cyan lines. (B) Process of capillary pressure signal: (i) Evolution of dimensionless capillary pressure calculated as the local curvature $P_{c}^{*}=1 / r^{*}$ in terms of number of step. Red circle marks the critical capillary pressure, where the pressure-driven advancement occurs. Yellow crosses represent the consequent spontaneous events (relaxation). (ii) Conversion from step into time expressed in terms of injected fluid area $A_{\mathrm{inj}}^{*}$ which is normalized by the average pore area. The time for Haines jump is regarded as instantaneous, i.e., only the start and end of $P_{c}^{*}$ are "felt" at the inlet. The $P_{c}^{*}$ values at same invasion progress are marked by the same number in (i) and (ii). (iii) Capillary pressure signal during the whole simulation. The black box is (ii). 
A
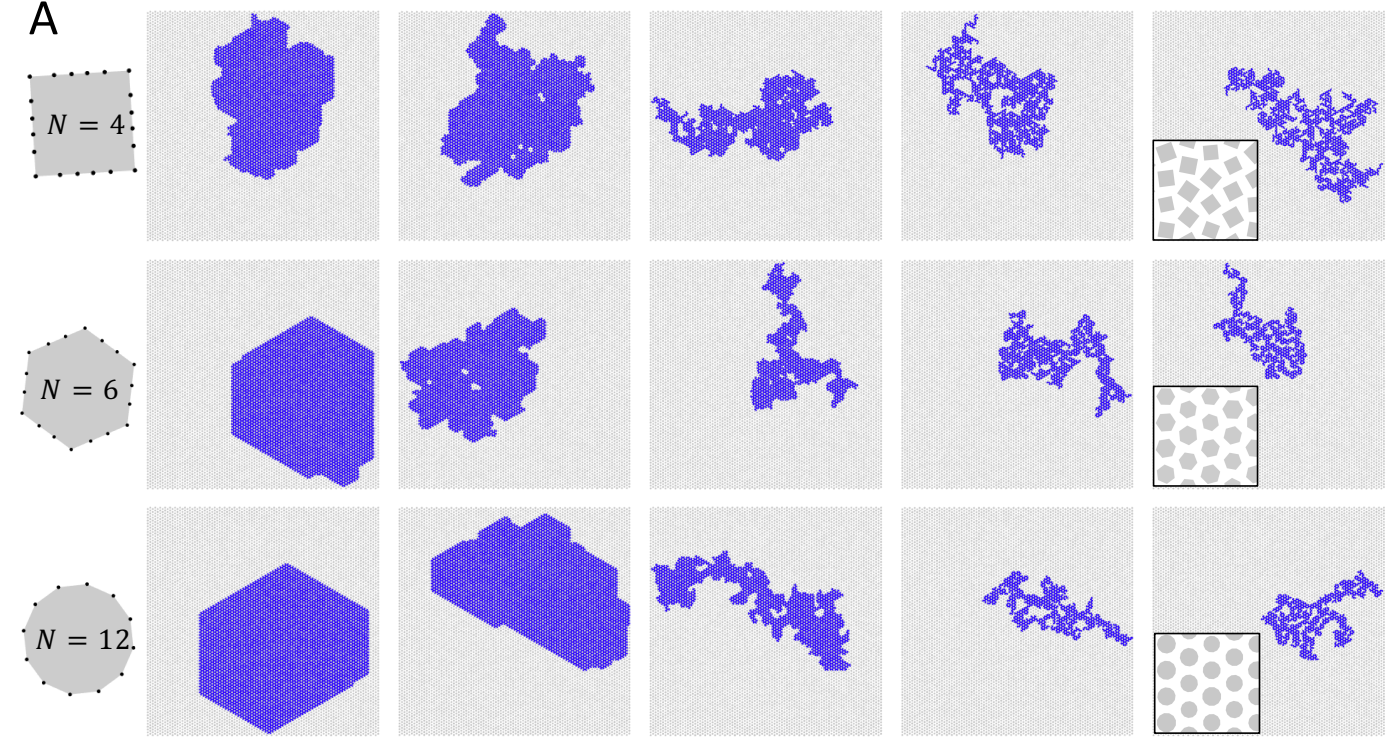

$\theta_{0}=45^{\circ}$

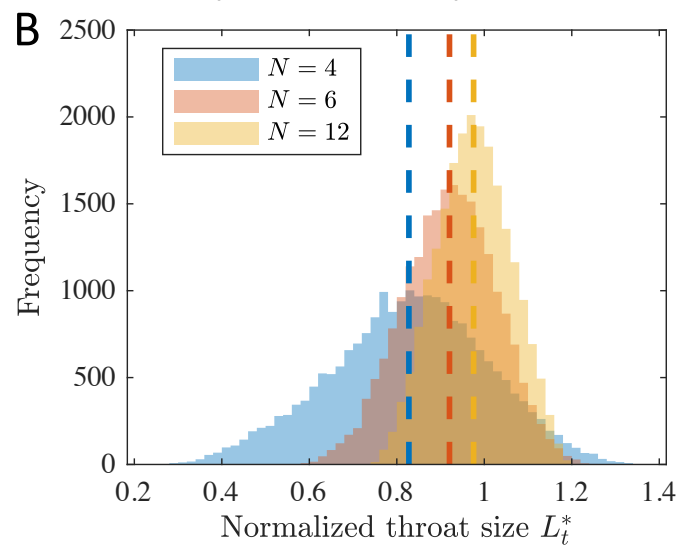

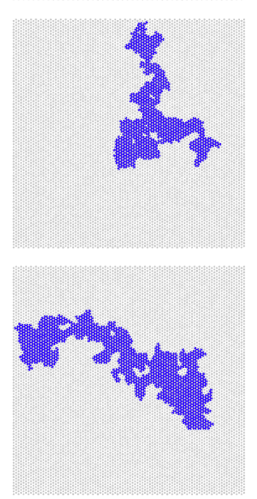

$\theta_{0}=90^{\circ}$

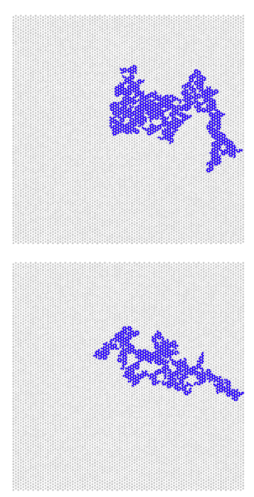

$\theta_{0}=120^{\circ}$

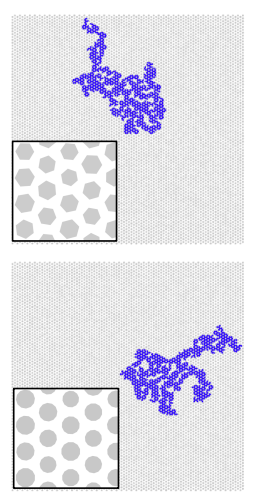

$\theta_{0}=150^{\circ}$

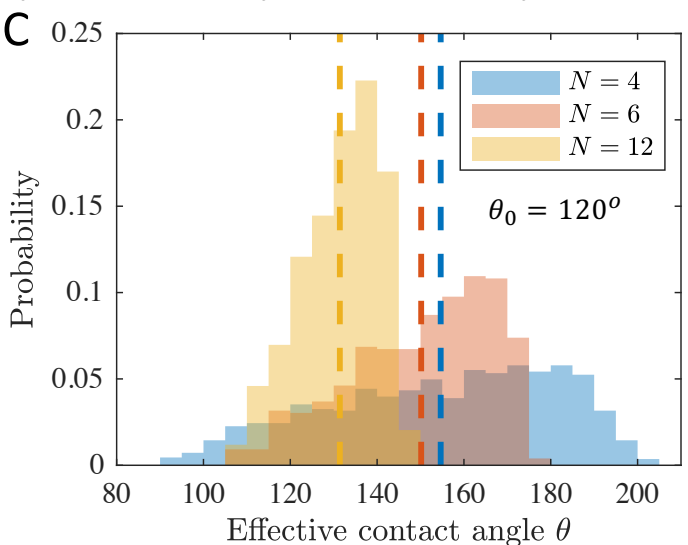

Figure 4. (A) Displacement patterns at the end of the simulation for different wettability conditions and grain shapes. Blue color represents the invading fluid injected from the center of domain. Grey color represents grains. Insets in the last column show the typical grain arrangement of a zoomed region. The simulation ends when the invading fluid reaches the boundary. (B) Distribution of normalized throat size for a typical set of porous media of different grain shapes, calculated as the shortest distance between adjacent grains divided by the throat size of volume equivalent spheres. (C) Contact angle distribution of one typical simulation at the end of simulation with an intrinsic contact angle $\theta_{0}=120^{\circ}$ for different grain shapes. The dashed lines marks the average value for the corresponding data. 

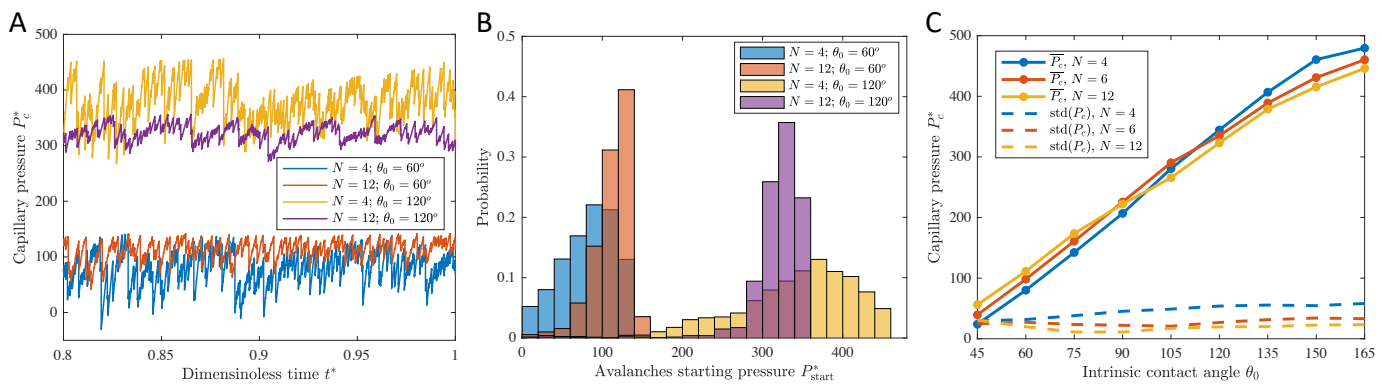

Figure 5. (A) Capillary pressure signals $P_{c}^{*}$ for media with squares and dodecagons at $\theta_{0}=60^{\circ}$ and $\theta_{0}=120^{\circ}$. Only last 20 percent of invasion is plotted for visualization purpose. (B) Corresponding avalanches starting pressure $P_{\text {start }}^{*}$ distribution for cases in (A), which is the $P_{c}^{*}$ at pressure-driven event (red circle in Fig. 3(B-i)). (C) Mean and standard deviation in $P_{c}^{*}$ for all grain shapes and wettability conditions. Values are calculated from five individual simulations.

invasion front becomes more irregular, indicating a shift of the dominance of local pore geometry from lattice structure towards grain shape. The results at $\theta_{0}=60^{\circ}$ demonstrate similar trend, with trapping events starting to occur for angular grains. With the increase of $\theta_{0}$, the displacement patterns experience a transition from compact displacement to capillary fingering. The distribution of the normalized throat size $L_{t}^{*}$, calculated as the shortest distance between adjacent grains divided by the throat size of the volumeequivalent spheres, is shown in Fig. 4(B). Although the media have similar grain size distribution (10 percent variation with uniform distribution) and arrangement (placed on triangular lattice), the $L_{t}^{*}$ distribution varies drastically for different grain shapes, with wider span for more angular grains, similar to the effect from increasing topological disorder (Wang et al., 2019). Another influence of particle shape is the smaller average throat size as angularity increases, despite almost constant porosity. For media filled with dodecagons, the average throat size is close to 1, i.e., the average throat size is similar to media with perfect spheres, which implies that the shape of dodecagons can be regarded as very close to spherical particles. Fig. $4(\mathrm{C})$ shows the effective contact angle distribution $\theta$ at the end of displacement (after the final relaxation process when invasion front reaches the boundary) for the case with an intrinsic contact angle $\theta_{0}=120^{\circ}$. Note that there is one effective contact angle per triple line (and thus two per meniscus). Due to the sharp edge pinning effect, the distribution narrows for grains with decreasing angularity. In the case of perfect spheres, one can expect a single value of $\theta=\theta_{0}$ according to Eqn. (1). Therefore, Fig. 4(B) and Fig. 4(C) summarize the important influences of particle angularity on pore geometry features and contact angle distribution, which will consequently impact the capillary pressure signal and invasion morphology.

\subsection{Capillary Pressure Signal}

As indicated in Fig. 5(A), the evolution of dimensionless capillary pressure $P_{c}^{*}$ shows larger fluctuations for media with squares compared with more spherical grains (dodecagons), implying greater randomness in local capillary resistance. At the same time, larger mean value is observed with increasing $\theta_{0}$, which is a direct result from greater curvature of menisci. The distribution of $P_{\text {start }}^{*}$, the avalanches starting pressure, can also be directly obtained from the simulation (Fig. 5(B)), where $P_{\text {start }}^{*}$ is the capillary pressure at pressuredriven event (red circle in Fig. 3(B-i)). For experiments conducted using spherical glass beads in drainage, $P_{\text {start }}^{*}$ is found to distribute within a relatively narrow region (Måløy et al., 1992; Furuberg et al., 1996; Moura et al., 2020). The $P_{\text {start }}^{*}$ distribution is linked 

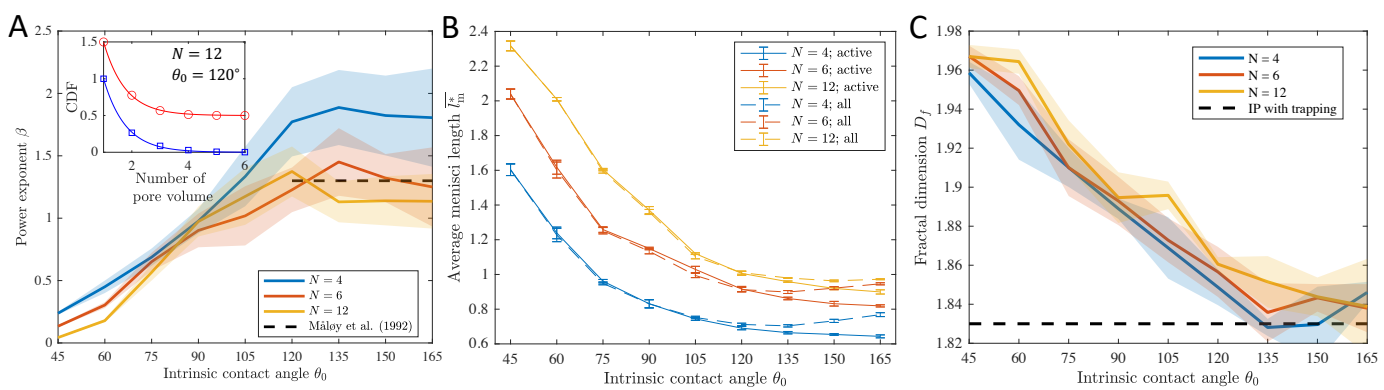

Figure 6. (A) Power exponent $\beta$ for different grain shapes and wetting conditions. The black-dashed line is from Måløy et al. (Måløy et al., 1992) in drainage experiment with glass beads (since the contact angle is not reported, it is assumed that $\theta_{0}>120^{\circ}$ ). Inset: cumulative Haines jump sizes (blue squares) and intervals (red circles) distribution for a typical simulation at $N=12$ with $\theta_{0}=120^{\circ}$. The intervals distribution is shifted by 0.5 for visualization purpose. Lines represent exponential fitting with 1.29 and 1.30 for jump sizes and interval sizes, respectively. (B) The normalized average meniscus width $\overline{l_{\mathrm{m}}^{*}}$ as a function of intrinsic contact angle. Solid (dashed) lines represent values calculated from active (all) menisci. (C) Fractal dimension calculated using box counting method as a function of intrinsic contact angle, with shaded area showing the standard deviation of five simulations.

to the total volume capacitance stored in all active menisci (Furuberg et al., 1996), which reflects the characteristics of pore geometry. For spherical grains with given packing structure, the capillary resistance is distinct between "pore" and "throat", leading to the fact that the avalanches are likely to initialize (and finish) at same location and consequently similar $P_{\text {start }}^{*}$. As the shape of particle becomes more angular, the distribution of $P_{\text {start }}^{*}$ widens as a result of increased impact from random orientations of grains and pinning of menisci, which is clearly demonstrated in Fig. 5(B). Fig. 5(C) depicts the mean and standard deviation of $P_{c}^{*}$ for all grain shapes and wettability with each value calculated from five individual simulations. As expected, the average capillary pressure increases with increasing contact angle, and the fluctuations in capillary pressure are found to be larger for more angular grains across all wetting conditions. Besides, it can be observed that $\overline{P_{c}^{*}}$ is greater (smaller) for angular grains in drainage (imbibition) conditions, with a crossover at around $\theta_{0} \approx 90^{\circ}$, which can be attributed to the change in pore and throat size distribution. It is well understood that the throat (pore) size controls the invasion process in drainage (imbibition). Fig. $4(\mathrm{C})$ indicates that greater angularity leads to smaller average throat size, and in the meantime greater average pore size (as the porosity is the same), which explains the observed higher capillary pressure in drainage and lower capillary pressure in imbibition.

\subsection{Haines Jumps and Patterns Characteristics}

The size of Haines jump can be obtained as the area filled between two pressuredriven events (shaded area in Fig. 1(D)), where filling events of single and multiple pores are observed. Both the cumulative pressure jump sizes and intervals distributions during drainage experiments have been found to follow an exponential law (Måløy et al., 1992; Furuberg et al., 1996), which is consistent with the simulation results (inset in Fig. 6A). The time interval between two jumps is expressed as the injected area of invading fluid, and the area of invading fluid is expressed in terms of number of average pore volume, calculated as the total pore space divided by the number of pores. Note that the cumulative intervals distribution is shifted upwards by 0.5 for visualization purpose. The power exponent $\beta$, which is regarded as a signature of the displacement process (Måløy et al., 
1992; Furuberg et al., 1996), is plotted for different grain shapes and wetting conditions (Fig. 6A). It can be seen that $\beta$ increases with the intrinsic contact angle, reaching a plateau at around $\theta_{0}=120^{\circ}$. Also, in general, the angularity positively correlates with the power exponent. The power exponent $\beta$ from Måløy et al. (Måløy et al., 1992) in drainage experiment with glass beads is added as black-dashed line for comparison. Since the contact angle was not reported, it is assumed that $\theta_{0}>120^{\circ}$. Their value of $\beta$ is close to the less angular grains (hexagons and dodecagons), which is consistent with the fact that glass beads are comparatively round and smooth.

The average meniscus width $\overline{l_{\mathrm{m}}^{*}}$ (or the average throat size where menisci are present), normalized by the throat size of porous medium of same porosity filled with mono-dispersed spheres, can reveal the distribution of menisci sizes for different particle shapes and wetting conditions. Fig. 6(B) shows $\overline{l_{\mathrm{m}}^{*}}$ as a function of intrinsic contact angle for different angularities with or without consideration of menisci belonging to trapped region. For all active menisci (solid lines), $\overline{l_{\mathrm{m}}^{*}}$ decreases with increasing $\theta_{0}$, reflecting stronger stability of pinned meniscus at small throat. Besides, despite constant porosity for all simulations, the average meniscus size is found to be smaller in angular grains as a result of (i) wider distribution of throat sizes (Fig. 4(B)), and (ii) greater capacity of pinning (upper bound in Eqn. (1)) as the local corner becomes sharper, leading to wider distribution of effective contact angles (Fig. 4(C)). If both active and inactive menisci are considered, however, a non-monotonic relationship is observed. This is a result of incompressibility of the trapped ganglia that prevent the menisci from further advancement and ultimately being pinned at narrower throats. Due to increased amount of trapping at larger contact angle (see Fig. 4(A)), this effect becomes significant at extreme nonwetting condition that leads to increase in $\overline{l_{\mathrm{m}}^{*}}$. This also implies that the pressure within the trapped ganglia could be lower than the capillary pressure signal measured at the inlet.

To quantify the displacement patterns, the fractal dimension $D_{f}$, as a measurement of the degree to which a pattern fills space, is calculated using box counting method. Fig. 6(C) demonstrates the transition from stable displacement with a $D_{f}$ of around 1.96 towards the regime of capillary fingering with $D_{f} \approx 1.84$, which is consistent with previously documented values of 1.96 and 1.83 for compact growth and invasion percolation, respectively (Wilkinson \& Willemsen, 1983; Lenormand \& Zarcone, 1989; Trojer et al., 2015; Zhao et al., 2016; M. J. Blunt, 2017; Primkulov et al., 2018). Furthermore, in spite of considerable variation (standard deviation, represented by the shaded area), an early transition towards capillary fingering, i.e., smaller $D_{f}$ at the same $\theta_{0}$, can be observed for grains with greater angularity, which confirms the qualitative observation in the displacement patterns in Fig.4(A). This could be partially explained by, apart from the variation in local pore structure, the increase of average effective contact angles in angular grains assemblies due to sharp edge pinning effect, which is evident in Fig. 4(C).

In this study, though we considered simplified particle shapes (regular polygons), more general and complex shapes can be easily implemented by changing the coordinates of grain vertices and updating the local corner angles accordingly. Also, it is possible to consider the viscous effect by incorporating, for example, the recently proposed moving capacitor model (Primkulov et al., 2019). Furthermore, in the current work the change in distribution of effective contact angle and throat size have been regarded as a direct result of variation in particle shape (Fig. 4(B) and Fig. 4(C)), as simultaneous variations in these two quantities, under fixed porosity, are inevitable with change in particle shape. It could be interesting to solely look at the impact of menisci pinning phenomena at sharp edges by excluding the change in throat (or pore) size distribution, although this would lead to different overall porosity of samples. Note that, for the choice of particle shapes investigated in this study, square grain is chosen as the most angular case instead of triangle. This is because that with current porosity 0.5912 , there will be overlaps of grains, if the shape is triangle, when they are randomly rotated. Since the porosity is already 
relatively large and we do not want to further increase it, also, we try to avoid manually specifying extra criterion during media generation and ensure fully random (homogeneous and isotropic) porous media, the most angular shape for current grain arrangement and porosity without grain overlap is square. On the other hand, dodecagon is used to represent circular grain, as the algorithm is not capable of doing perfect circles since the calculation of critical capillary pressure and advancement of menisci is based on discretization of the grains, and perfect circles correspond to infinite number of mesh points. Nevertheless, it was shown that the case for dodecagon is close to circle, as the average throat size is very close to the porous media filled with perfect circles (Fig. 4(B)).

\section{Conclusions}

In conclusion, we presented an extended pore-network model (EPONM) to probe the effect of grain shapes on quasi-static fluid-fluid displacement in porous media. The model incorporates the mechanisms of pore-scale instabilities (Cieplak \& Robbins, 1988, 1990), volume capacitance model (Måløy et al., 1992; Furuberg et al., 1996), and sharp edge pinning effect (Gibbs, 1961; Oliver et al., 1977). This allows us to reproduce the multiphase flow patterns across a wide range of wetting conditions for different grain shapes. The algorithm, with further extension, i.e., mainly on geometry description and generalization on determination of instability modes, should be applicable to porous media with arbitrary grain shape/location, such as reconstructed pore geometry from 2D scan of rocks, offering a rigorous approach for investigation of how topological features modify the multiphase displacement in porous media.

At the pore scale, increase in grain angularity not only introduces greater heterogeneity in pore geometry, but also amplify the effect of menisci pinning at corners. This is directly reflected by the variations in distributions of throat sizes (Fig. 4(B)) and effective contact angles (Fig. 4(C)), which consequently impact both the mean value and fluctuation of the capillary pressure signal (Fig. 5(A)). Macroscopically, an earlier transition from stable displacement towards the regime of capillary fingering is observed both qualitatively from the invasion morphology (Fig. 4(A)) and quantitatively as indicated by the fractal dimension (Fig. 6(C)). Various characteristic metrics have been calculated for comparison with past experimental works, including the distribution of avalanches starting pressure, Haines jump size and interval. Reasonable agreement is observed, and impacts of grain shape are discussed. In particular, under the condition of same porosity for all studied cases, the average size of menisci is found to be smaller in porous media with angular grains, showing a tendency of pinning at narrower throats as a result of wider distribution of throat sizes (Fig. 4(B)) and greater pinning strength (Eqn. (1)).

Our results have provided independent corroboration of wide distribution of contact angles observed experimentally in mineralogically homogeneous porous media. The profound influences of grain shape are highlighted by systematically analyzing the displacement processes, deepening the understanding of the interplay between pore geometry and wettability. The proposed pore-network model offers an efficient approach for investigation of multiphase flow in natural porous media.

\section{Acknowledgments}

This work was financially supported by Australian Research Council (Projects DP170102886) and The University of Sydney SOAR Fellowship. YG acknowledges the financial support of Labex MMCD(ANR-11-LABX-022-01) for his stay at Laboratoire Navier at ENPC. ZW thanks Ms Zi Ying for fruitful discussion. Data sets associated with this work are available online (https://doi.org/10.6084/m9.figshare.13369733). 


\section{References}

AlRatrout, A., Blunt, M. J., \& Bijeljic, B. (2018). Wettability in complex porous materials, the mixed-wet state, and its relationship to surface roughness. Proceedings of the National Academy of Sciences, 115(36), 8901-8906. doi: 10.1073/pnas .1803734115

Armstrong, R. T., Georgiadis, A., Ott, H., Klemin, D., \& Berg, S. (2014). Critical capillary number: Desaturation studied with fast x-ray computed microtomography. Geophysical Research Letters, 41(1), 55-60. doi: 10.1002/2013GL058075

Blunt, M., Fayers, F., \& Orr, F. M. (1993). Carbon dioxide in enhanced oil recovery. Energy Conversion and Management, 34(9), 1197 - 1204. (Proceedings of the International Energy Agency Carbon Dioxide Disposal Symposium)

Blunt, M. J. (1998). Physically-based network modeling of multiphase flow in intermediate-wet porous media. Journal of Petroleum Science and Engineering, 20 (3), 117 - 125. doi: https://doi.org/10.1016/S0920-4105(98)00010-2

Blunt, M. J. (2001). $\quad$ Flow in porous media - pore-network models and multiphase flow. Current Opinion in Colloid ES Interface Science, 6(3), 197 - 207. Retrieved from http://www.sciencedirect.com/science/article/pii/ S135902940100084X doi: https://doi.org/10.1016/S1359-0294(01)00084-X

Blunt, M. J. (2017). Multiphase flow in permeable media: A pore-scale perspective. Cambridge University Press. doi: 10.1017/9781316145098

Blunt, M. J., Alhosani, A., Lin, Q., Scanziani, A., \& Bijeljic, B. $\quad$ (2021). Determination of contact angles for three-phase flow in porous media using an energy balance. Journal of Colloid and Interface Science, 582, 283 - $290 . \quad$ doi: https://doi.org/10.1016/j.jcis.2020.07.152

Blunt, M. J., Lin, Q., Akai, T., \& Bijeljic, B. (2019). A thermodynamically consistent characterization of wettability in porous media using highresolution imaging. Journal of Colloid and Interface Science, 552, 59 - 65. doi: https://doi.org/10.1016/j.jcis.2019.05.026

Cassie, A. B. D., \& Baxter, S. (1944). Wettability of porous surfaces. Trans. Faraday Soc., 40, 546-551. Retrieved from http://dx.doi.org/10.1039/ TF9444000546 doi: 10.1039 /TF9444000546

Chapuis, O., Prat, M., Quintard, M., Chane-Kane, E., Guillot, O., \& Mayer, N. (2008). Two-phase flow and evaporation in model fibrous media: Application to the gas diffusion layer of pem fuel cells. Journal of Power Sources, 178(1), 258268. Retrieved from https://www.sciencedirect.com/science/article/pii/ S0378775307026523 doi: https://doi.org/10.1016/j.jpowsour.2007.12.011

Chen, Y.-F., Wu, D.-S., Fang, S., \& Hu, R. (2018). Experimental study on twophase flow in rough fracture: Phase diagram and localized flow channel. International Journal of Heat and Mass Transfer, 122, 1298 - 1307. doi: https://doi.org/ 10.1016/j.ijheatmasstransfer.2018.02.031

Cieplak, M., \& Robbins, M. O. (1988, May). Dynamical transition in quasistatic fluid invasion in porous media. Phys. Rev. Lett., 60, 2042-2045. doi: 10.1103/ PhysRevLett.60.2042

Cieplak, M., \& Robbins, M. O. (1990, Jun). Influence of contact angle on quasistatic fluid invasion of porous media. Phys. Rev. B, 41,11508-11521. doi: 10.1103/ PhysRevB.41.11508

Crisp, D. J., \& Thorpe, W. H. (1948). The water-protecting properties of insect hairs. Discuss. Faraday Soc., 3, 210-220. Retrieved from http://dx.doi.org/10 .1039/DF9480300210 doi: 10.1039/DF9480300210

Furuberg, L., Måløy, K. J., \& Feder, J. (1996, Jan). Intermittent behavior in slow drainage. Phys. Rev. E, 53, 966-977. doi: 10.1103/PhysRevE.53.966

Gao, N., \& Yan, Y. (2009). Modeling superhydrophobic contact angles and wetting transition. Journal of Bionic Engineering, 6(4), 335-340. Retrieved from 
http://www.sciencedirect.com/science/article/pii/S1672652908601353 doi: https://doi.org/10.1016/S1672-6529(08)60135-3

Geistlinger, H., \& Zulfiqar, B. (n.d.). The impact of wettability and surface roughness on fluid displacement and capillary trapping in $2 \mathrm{~d}$ - and $3 \mathrm{~d}$-porous media part 1: Wettability-controlled phase transition of trapping efficiency in glass beads packs. Water Resources Research, n/a(n/a), e2019WR026826. (e2019WR026826 2019WR026826) doi: 10.1029/2019WR026826

Gibbs, J. W. (1961). The scientific papers (Vol. 1). New York: Dover Publications.

Holtzman, R. (2016). Effects of pore-scale disorder on fluid displacement in partially-wettable porous media [Journal Article]. Sci Rep, 6, 36221. doi: $10.1038 /$ srep36221

Holtzman, R., \& Segre, E. (2015, Oct). Wettability stabilizes fluid invasion into porous media via nonlocal, cooperative pore filling. Phys. Rev. Lett., 115, 164501. doi: 10.1103/PhysRevLett.115.164501

Hu, R., Lan, T., Wei, G.-J., \& Chen, Y.-F. (2019). Phase diagram of quasi-static immiscible displacement in disordered porous media. Journal of Fluid Mechanics, 875, 448-475. doi: $10.1017 / \mathrm{jfm} .2019 .504$

Ju, Y., Gong, W., Chang, W., \& Sun, M. (2020). Effects of pore characteristics on water-oil two-phase displacement in non-homogeneous pore structures: A pore-scale lattice boltzmann model considering various fluid density ratios. International Journal of Engineering Science, 154, $103343 . \quad$ doi: https://doi.org/10.1016/j.ijengsci.2020.103343

Jung, M., Brinkmann, M., Seemann, R., Hiller, T., Sanchez de La Lama, M., \& Herminghaus, S. (2016, Nov). Wettability controls slow immiscible displacement through local interfacial instabilities. Phys. Rev. Fluids, 1, 074202. doi: 10.1103/PhysRevFluids.1.074202

Lake, L. W., Johns, R., Rossen, B., \& Pope, G. (2014). Fundamentals of enhanced oil recovery. Society of Petroleum Engineers.

Lenormand, R. (1990, dec). Liquids in porous media. Journal of Physics: Condensed Matter, 2(S), SA79-SA88. Retrieved from https://doi.org/10.1088/ 0953-8984/2/s/008 doi: 10.1088/0953-8984/2/s/008

Lenormand, R., Touboul, E., \& Zarcone, C. (1988). Numerical models and experiments on immiscible displacements in porous media [Journal Article]. Journal of Fluid Mechanics, 189(165-187). doi: 10.1017/s0022112088000953

Lenormand, R., \& Zarcone, C. $\quad$ (1989, Dec 01). Capillary fingering: Percolation and fractal dimension. Transport in Porous Media, 4(6), 599-612. doi: 10.1007/ BF00223630

Lipiec, J., Kuś, J., Słlowińska-Jurkiewicz, A., \& Nosalewicz, A. (2006). Soil porosity and water infiltration as influenced by tillage methods. Soil and Tillage Research, 89(2), 210 - 220. doi: https://doi.org/10.1016/j.still.2005.07.012

Måløy, K. J., Furuberg, L., Feder, J., \& Jøssang, T. （1992, Apr). Dynamics of slow drainage in porous media. Phys. Rev. Lett., 68, 2161-2164. Retrieved from https://link.aps.org/doi/10.1103/PhysRevLett.68.2161 doi: 10.1103/PhysRevLett.68.2161

Marmur, A. (2003). Wetting on hydrophobic rough surfaces: To be heterogeneous or not to be? Langmuir, 19(20), 8343-8348. Retrieved from https://doi.org/10 .1021/la0344682 doi: 10.1021/la0344682

Mason, G., \& Morrow, N. R. (1994). Effect of contact angle on capillary displacement curvatures in pore throats formed by spheres. Journal of Colloid and Interface Science, 168(1), 130-141. Retrieved from https://www.sciencedirect.com/ science/article/pii/S0021979784714020 doi: https://doi.org/10.1006/ jcis.1994.1402

Matter, J. M., Stute, M., Snæbjörnsdottir, S. Ó., Oelkers, E. H., Gislason, S. R., Aradottir, E. S., ... Broecker, W. S. (2016). Rapid carbon mineralization for per- 
manent disposal of anthropogenic carbon dioxide emissions. Science, 352(6291), 1312-1314. doi: 10.1126/science.aad8132

Mehmani, A., Kelly, S., Torres-Verdín, C., \& Balhoff, M. ～(2019). Residual oil saturation following gas injection in sandstones: Microfluidic quantification of the impact of pore-scale surface roughness. Fuel, 251, 147-161. doi: https://doi.org/10.1016/j.fuel.2019.02.118

Mishra, H., Schrader, A. M., Lee, D. W., Gallo, A., Chen, S.-Y., Kaufman, Y., ... Israelachvili, J. N. (2016). Time-dependent wetting behavior of pdms surfaces with bioinspired, hierarchical structures. ACS Applied Materials \& Interfaces, 8(12), 8168-8174. Retrieved from https://doi.org/10.1021/acsami.5b10721 doi: 10.1021 /acsami.5b10721

Moulinet, S., \& Bartolo, D. (2007, Nov 01). Life and death of a fakir droplet: Impalement transitions on superhydrophobic surfaces. The European Physical Journal E, 24(3), 251-260. Retrieved from https://doi.org/10.1140/epje/i2007 -10235-y doi: 10.1140/epje/i2007-10235-y

Moura, M., Måløy, K. J., Flekkøy, E. G., \& Toussaint, R. (2020). Intermittent dynamics of slow drainage experiments in porous media: Characterization under different boundary conditions. Frontiers in Physics, 7, 217. Retrieved from https://www.frontiersin.org/article/10.3389/fphy.2019.00217 doi: 10.3389/fphy.2019.00217

Nadim, F., Hoag, G. E., Liu, S., Carley, R. J., \& Zack, P. (2000). Detection and remediation of soil and aquifer systems contaminated with petroleum products: an overview. Journal of Petroleum Science and Engineering, 26(1), 169 - 178.

Oliver, J., Huh, C., \& Mason, S. (1977). Resistance to spreading of liquids by sharp edges. Journal of Colloid and Interface Science, 59(3), 568 - 581. Retrieved from http://www.sciencedirect.com/science/article/pii/0021979777900522 doi: https://doi.org/10.1016/0021-9797(77)90052-2

Papadopoulos, P., Mammen, L., Deng, X., Vollmer, D., \& Butt, H.-J. (2013). How superhydrophobicity breaks down. Proceedings of the National Academy of Sciences, 110(9), 3254-3258. Retrieved from https://www.pnas.org/content/110/ 9/3254 doi: $10.1073 /$ pnas.1218673110

Primkulov, B. K., Pahlavan, A. A., Fu, X., Zhao, B., MacMinn, C. W., \& Juanes, R. (2019). Signatures of fluid-fluid displacement in porous media: wettability, patterns and pressures. Journal of Fluid Mechanics, 875, R4. doi: $10.1017 /$ jfm.2019.554

Primkulov, B. K., Talman, S., Khaleghi, K., Rangriz Shokri, A., Chalaturnyk, R., Zhao, B., ... Juanes, R. (2018, Oct). Quasistatic fluid-fluid displacement in porous media: Invasion-percolation through a wetting transition. Phys. Rev. Fluids, 3, 104001. doi: 10.1103/PhysRevFluids.3.104001

Purcell, W. (1950, 08). Interpretation of Capillary Pressure Data. Journal of Petroleum Technology, 2(08), 11-12. Retrieved from https://doi.org/10.2118/ 950369-G doi: 10.2118/950369-G

Rabbani, H. S., Or, D., Liu, Y., Lai, C.-Y., Lu, N. B., Datta, S. S., .. Shokri, N. (2018). Suppressing viscous fingering in structured porous media. Proceedings of the National Academy of Sciences, 115, 4833-4838. doi: 10.1073/ pnas. 1800729115

Ran, H., Jiamin, W., Zhibing, Y., Yi-Feng, C., \& Tetsu, T. (2018). Wettability and flow rate impacts on immiscible displacement: A theoretical model. Geophysical Research Letters, 45(7), 3077-3086. doi: 10.1002/2017GL076600

Sbragaglia, M., Peters, A. M., Pirat, C., Borkent, B. M., Lammertink, R. G. H., Wessling, M., \& Lohse, D. (2007, Oct). Spontaneous breakdown of superhydrophobicity. Phys. Rev. Lett., 99, 156001. Retrieved from https:// link.aps.org/doi/10.1103/PhysRevLett.99.156001 doi: 10.1103/ PhysRevLett.99.156001 
Seo, D., Schrader, A. M., Chen, S.-Y., Kaufman, Y., Cristiani, T. R., Page, S. H., ... Israelachvili, J. N. (2018). Rates of cavity filling by liquids. Proceedings of the National Academy of Sciences, 115(32), 8070-8075. Retrieved from https://www.pnas.org/content/115/32/8070 doi: 10.1073/pnas.1804437115

Szulczewski, M. L., MacMinn, C. W., Herzog, H. J., \& Juanes, R. (2012). Lifetime of carbon capture and storage as a climate-change mitigation technology. Proceedings of the National Academy of Sciences, 109(14), 5185-5189. doi: 10.1073/pnas .1115347109

Trojer, M., Szulczewski, M. L., \& Juanes, R. (2015, May). Stabilizing fluid-fluid displacements in porous media through wettability alteration. Phys. Rev. Applied, 3, 054008. doi: 10.1103/PhysRevApplied.3.054008

Wang, Z., Chauhan, K., Pereira, J.-M., \& Gan, Y. (2019, Mar). Disorder characterization of porous media and its effect on fluid displacement. Phys. Rev. Fluids, 4, 034305. doi: 10.1103/PhysRevFluids.4.034305

Wang, Z., Pereira, J.-M., \& Gan, Y. (2020). Effect of wetting transition during multiphase displacement in porous media. Langmuir, 36 (9), 2449-2458. (PMID: 32070092) doi: 10.1021/acs.langmuir.9b03780

Wenzel, R. N. (1936). Resistance of solid surfaces to wetting by water. Industrial \&3 Engineering Chemistry, 28(8), 988-994. Retrieved from https://doi.org/ 10.1021/ie50320a024 doi: 10.1021/ie50320a024

Wilkinson, D., \& Willemsen, J. F. (1983, oct). Invasion percolation: a new form of percolation theory. Journal of Physics A: Mathematical and General, 16(14), $3365-3376$. Retrieved from https://doi.org/10.1088\%2F0305-4470\%2F $16 \% 2 \mathrm{~F} 14 \%$ 2F028 doi: $10.1088 / 0305-4470 / 16 / 14 / 028$

Xu, W., Ok, J. T., Xiao, F., Neeves, K. B., \& Yin, X. (2014). $\quad$ Effect of pore geometry and interfacial tension on water-oil displacement efficiency in oil-wet microfluidic porous media analogs. Physics of Fluids, 26(9), 093102. doi: $10.1063 / 1.4894071$

Yortsos, Y. C., Xu, B., \& Salin, D. $\quad$ (1997, Dec). Phase diagram of fully developed drainage in porous media. Phys. Rev. Lett., 79, 4581-4584. doi: 10.1103/PhysRevLett.79.4581

Zhao, B., MacMinn, C. W., \& Juanes, R. (2016). Wettability control on multiphase flow in patterned microfluidics. Proceedings of the National Academy of Sciences, 113(37), 10251-10256. doi: 10.1073/pnas.1603387113

Zulfiqar, B., Vogel, H., Ding, Y., Golmohammadi, S., Küchler, M., Reuter, D., \& Geistlinger, H. (n.d.). The impact of wettability and surface roughness on fluid displacement and capillary trapping in $2 \mathrm{~d}$ - and $3 \mathrm{~d}$-porous media: Part 2: Combined effect of wettability, surface roughness, and pore space structure on trapping efficiency in sand packs and micromodels. Water Resources Research, $n / a(\mathrm{n} / \mathrm{a}), \mathrm{e} 2020 \mathrm{WR} 027965 . \quad$ (e2020WR027965 2020WR027965) doi: 10.1029/2020WR027965 